\title{
Electronic transport in B-N substituted bilayer graphene nanojunctions
}

\author{
Daniele Giofré, ${ }^{1, *}$ Davide Ceresoli, ${ }^{2}$ Guido Fratesi,${ }^{3}$ and Mario I. Trioni ${ }^{2, \dagger}$ \\ ${ }^{1}$ Dipartimento di Fisica, Università Milano-Bicocca, Piazza della Scienza 3, 20126 Milan, Italy \\ ${ }^{2}$ CNR - National Research Council of Italy, ISTM, via Golgi 19, 20133 Milan, Italy \\ ${ }^{3}$ Dipartimento di Fisica, Università di Milano, via Celoria 16, 20133 Milan, Italy \\ (Received 22 February 2015; revised manuscript received 21 April 2016; published 11 May 2016)
}

\begin{abstract}
We investigated a suspended bilayer graphene where the bottom/top layer is doped by boron/nitrogen substitutional atoms. By using density functional theory calculations, we found that at high dopant concentration (one B-N pair every $32 \mathrm{C}$ atoms), the electronic structure of the bilayer does not depend on the B-N distance but on the relative occupation of the bilayer graphene sublattices by $\mathrm{B}$ and $\mathrm{N}$. The presence of the dopants and the consequent charge transfer establish a built-in electric field between the layers, giving rise to an energy gap. We further investigated the electronic transport properties and found that intralayer current is weakly influenced by the presence of these dopants while the interlayer one is enhanced for biases, allowing an easy tunneling between layers. This effect leads to current rectification in asymmetric junctions.
\end{abstract}

DOI: 10.1103/PhysRevB.93.205420

Bilayer graphene (BLG) is a two-dimensional material constituted by two stacked graphene layers. It has recently attracted much interest because it shows exceptionally high charge mobility like in single-layer graphene [1]. Charge transport in BLG is tied to the in-plane direction of each of the two layers and it has been shown that a gate potential can induce an energy gap in bilayer graphene [2]. As a consequence, BLG has been proposed to be more suitable than single-layer graphene to realize carbon-based field-effect logic devices, as the new bilayer-graphene-based transistor [3-5]. However, due to the small density of states at the Fermi level of BLG, very large electric fields are needed in order to create an unbalance in the charge distribution. This has a deleterious impact on the performance and stability of gated BLG. For instance, graphene and BLG deposited on oxide dielectrics such as $\mathrm{SiO}_{2}$ show a large hysteresis in the $I-V$ curve, due to the charging/discharging of point defects in the oxide. Moreover, charged impurities in the gate oxide and at the graphene-gate interface have been shown to dramatically reduce the mobility in graphene-based devices [6,7]. On the other hand, the low density of states can be exploited to induce the effects of a large electric field in BLG. In fact, the addition or removal of even a small amount of charge from each layer causes an abrupt change of the Fermi level.

In this paper, we simulate a suspended bilayer graphene nanojunction where boron and nitrogen atoms substitute carbon atoms in the bottom and top layers, respectively. The equal number of $\mathrm{B}$ and $\mathrm{N}$ atoms assures that the system is isoelectronic to the pristine bilayer. By first-principles calculations, we find that a large built-in electric field is then established, due to the combination of two effects: the first is the Fermi-level mismatch between the isolated B-rich and N-rich layers, and the second is a partial charge transfer between them as they are put in contact. As a consequence, a small energy gap appears, enabling this system

\footnotetext{
${ }^{*}$ Present address: Laboratory of Computational Science and Modelling, Institute of Materials, Ecole Polytechnique Fédérale de Lausanne, Lausanne, Switzerland.

${ }^{\dagger}$ Corresponding author: mario.trioni@istm.cnr.it
}

to be employed for nanoelectronics applications. In view of this, we also investigate the ballistic electron transport in graphene nanojunctions containing $\mathrm{B}$ and $\mathrm{N}$ substitutional atoms and show the possibility of a rectification behavior that is independent of the relative sublattice occupations.

We performed density functional theory (DFT) calculations with the local-basis SIESTA code [8-11]. We used normconserving pseudopotentials [12] and the double- $\zeta$ polarized basis set. The exchange correlation potential is described by the Perdew-Burke-Ernzerhof (PBE) generalized gradient approximation (GGA) [13], and the weak van der Waals forces between the graphene layers are described by a classical $C / r^{6}$ term, parametrized by Grimme [14]. In this theoretical framework, we obtained an interlayer distance of $3.32 \AA$ for pristine BLG, in good agreement with the experimental value of $3.36 \AA$ [15]. In every subsequent calculation, we fixed the in-plane lattice constant to the calculated value of $2.474 \AA$, which is close to the experimental value of $2.46 \AA$ [16]. We make use of the slab-dipole correction to avoid spurious effects due to the built-in dipole in the graphene bilayer. Real-space grid and Brillouin-zone sampling are taken fully convergent.

We simulated the doping of each layer by constructing a $4 \times 4$ supercell, i.e., 32 carbon atoms per layer with a single B$\mathrm{N}$ substitutional pair. We recall that graphene is constituted by a bipartite lattice and we denote by S1 and S2 the first and second sublattice. In the case of BLG, the two sublattices are no more equivalent due to their Bernal stacking. The first sublattice of BLG (S1) is constituted by carbon atoms sitting above/below carbon atoms from the other layer. The second sublattice (S2) is constituted by carbon atoms positioned above/below the geometrical center of the hexagons from the other layer, as shown in Fig. 1.

The electronic properties of the system depend on the relative position of the two dopants in the graphene sublattices. In $\mathrm{B}-\mathrm{N}$ substituted BLG with one B-N pair, there are three nonhomologous configurations. The first is when $\mathrm{B}$ and $\mathrm{N}$ belong to different sublattices (A in Fig. 1); the second is characterized by $\mathrm{B}$ and $\mathrm{N}$ belonging to the $\mathrm{S} 1$ sublattices (in this case, they can be situated one on top of the other; see configuration B in Fig. 1); in the third configuration, B and $\mathrm{N}$ occupy the S2 sublattices (C in Fig. 1). In the $4 \times 4$ 

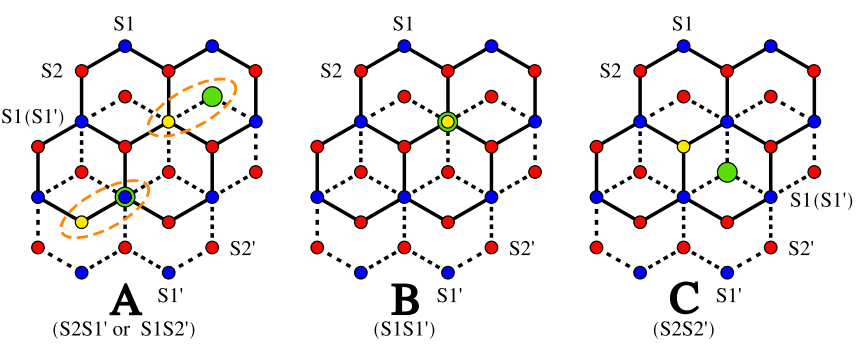

FIG. 1. Possible arrangements for a substitutional B-N pair in graphene bilayer. Yellow sphere: N; green sphere: B; blue and red spheres are $\mathrm{C}$ atoms of the two inequivalent sublattices $\mathrm{S} 1$ and $\mathrm{S} 2$, respectively.

supercell, additional arrangements are possible which differ from $\mathbf{A}, \mathbf{B}$, and $\mathbf{C}$ for the relative $\mathrm{B}-\mathrm{N}$ distance. We verified that for a given B-N substitution into the two sublattices, the distance between the impurities has a minor influence on the electronic properties of the system. This is reasonable because the presence of an impurity gives a perturbation which has a large spatial extent but is localized on the specific sublattice only.

The three configurations are almost isoenergetic, with $\mathbf{C}$ being the most stable one, and configurations $\mathbf{A}$ and $\mathbf{B}$ higher in energy by $\sim 0.07$ and $\sim 0.06 \mathrm{eV}$, respectively. In the following, we will concentrate on case $\mathbf{C}$, eventually verifying that the conclusions can be extended to the other cases.

In Fig. 2, we report the density of states (DOS) and the band structure of the $\mathbf{C}$ configuration. In the upper panel, the DOS show two interesting features. The first one is the appearance of an energy gap of $0.32 \mathrm{eV}$ comprising the Fermi level. The second one is the presence, in each layer, of the characteristic "V-shaped" DOS centered around the Dirac points, which are separated by an energy $U \sim 1.4 \mathrm{eV}$. This value can also be extracted from the band structure as the energy separation of the two Dirac points, which are located at K, and referring to each of the two doped layers. The value of $U$ can be rationalized in terms of the Fermi energy shift of the isolated doped graphene layers $(\sim \pm 0.8 \mathrm{eV})$, mitigated by $0.2 \mathrm{eV}$ due to the charge transfer occurring from the $\mathrm{N}$-doped layer to the B-doped one. The dipole moment generated by the redistribution of electronic charge is $2.77 \mathrm{D}$ per $\mathrm{B}-\mathrm{N}$ pair. Such a dipole creates a built-in electric field $E=0.03 \mathrm{~V} / \AA$ along the direction perpendicular to the sheets.

From the band structure reported in Fig. 2, we observe that the Kohn-Sham band gap is direct and located away from the $\mathrm{K}$ point, along the $\Gamma-\mathrm{K}$ path. One can also note that the highest occupied band belongs to the B-doped layer, while the lowest unoccupied one belongs to the $\mathrm{N}$-doped layer. This band structure shows two "mexican hats" centered around $\mathrm{K}$, like the band structure of BLG subject to a perpendicular electric field. In fact, by comparing to Fig. 2(c) of Ref. [17], our $U$ corresponds to the gap at K (named "eV" in Ref. [17]) and our band gap of $0.32 \mathrm{eV}$ corresponds to $\Delta_{g}$ in that work. This gap is only weakly dependent on the doping concentration (as an example, we compute a value of $0.29 \mathrm{eV}$ for a larger $7 \times 7$ unit cell). The difference of the electronic structures between the donor- and acceptor-doped graphene layers appears promising for application in nanoelectronics
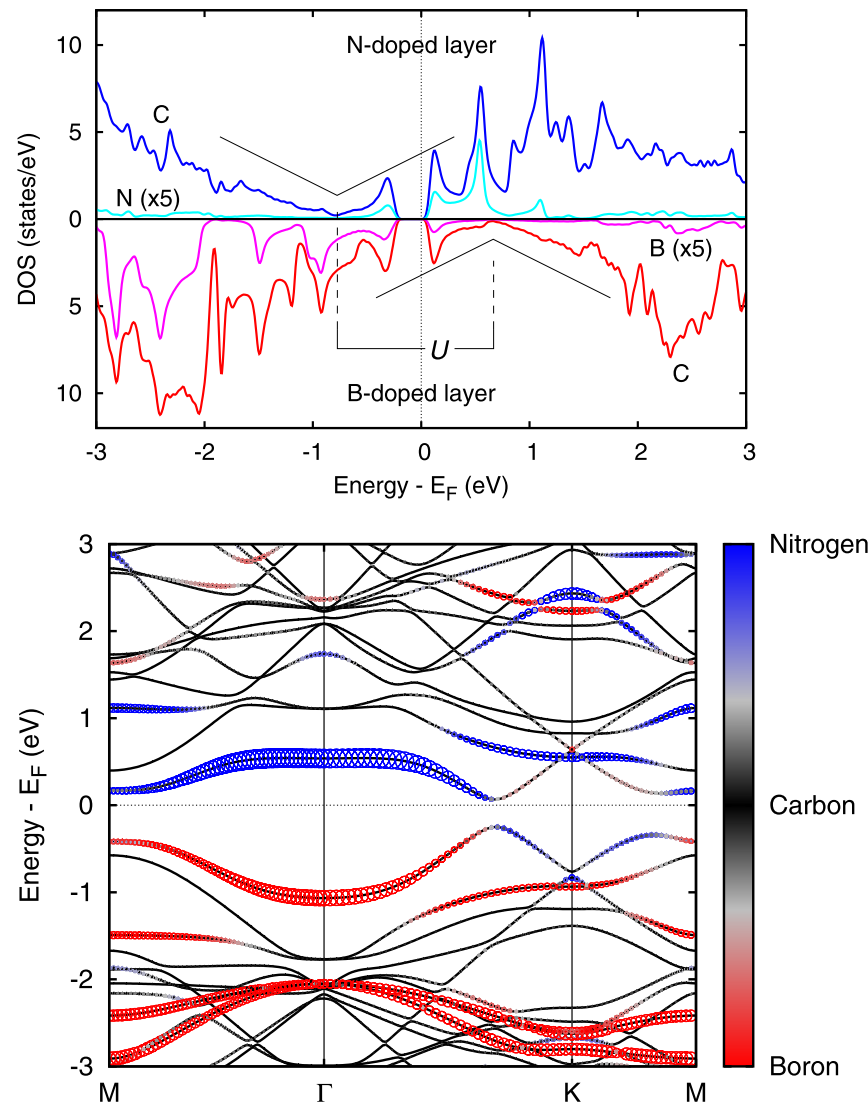

FIG. 2. Top panel: Density of states of the B-N doped BLG. The projected DOS on $\mathrm{N}$ and $\mathrm{C}$ atoms of the $\mathrm{N}$-doped graphene layer are reported in the upper part, while those projected on $\mathrm{B}$ and $\mathrm{C}$ atoms of the B-doped graphene layer are reported in the lower part. Bottom panel: band structure of B-N-doped BLG. The weight on B (red) and $\mathrm{N}$ (blue) is also reported. The size of the circles is proportional to the weight of the eigenstates on specific atoms.

and motivated us to study ballistic transport through B-Ndoped BLG, which is the dominant transport mechanism in these devices, since the mean free path is typically larger that the size of our nanojunction [18]. As shown in Ref. [19], even the presence of dopants does not introduce severe quantum interference effects. In fact, for such systems, new conduction channels allowing the interlayer transport can be available.

The first-principles quantum transport calculations were performed using the TransIESTA code [20], which employs the nonequilibrium Green's function (NEGF) formalism in conjunction with DFT. Within the NEGF-DFT approach, the system has been divided into three regions: the left and right electrodes, and the scattering region. The latter contains a portion of physical electrodes, the so-called right and left contacts, where all screening effects take place. Along the direction perpendicular to that of the current flow, all three regions are infinitely extended and periodically repeated. In order to apply an external bias, the Fermi levels of the electrodes are shifted relative to each other and the electronic occupations of the system are determined self-consistently using the left/right spectral function in the energy window fixed by the electrochemical potentials of electrodes. The transmission function is worked out in the Landauer-Büttiker 


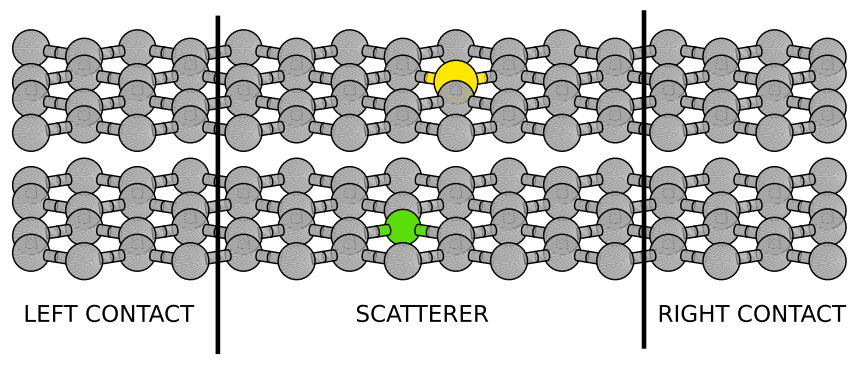

FIG. 3. Transport geometry of the overlay B-N-doped junction in the $\mathbf{C}$ arrangement. The upper layer contains the nitrogen atom while the lower layer contains the boron one.

approximation [21], which accounts for ballistic transport. We first consider the electron transport in BLG in the zigzag direction, through the B-N doped region in $\mathbf{C}$ configuration. We recall that the scattering region is finite along the transport direction but is periodically repeated along the transverse one so no unsaturated $\mathrm{C}$ edges are present (unlike the case of transport through nanoribbons). In this configuration, labeled as "overlay" and depicted in Fig. 3, the left and right electrodes are made of 32 atoms of pristine BLG while the scattering region contains a single pair of B-N dopants and 62 carbon atoms.

With respect to the infinite B-N-doped BLG discussed above, the presence of the electrodes modifies the electronic properties of this "open" system, mainly because the Fermi level is now fixed to that of the infinitely extended pristine BLG. Furthermore, the translational invariance along the transport direction is lost and the electronic states are now labeled by only the $k$ component parallel to the interface $\left(k_{\|}\right)$. The spectral properties can be observed in the $k_{\|}$-resolved DOS of the system, reported in Fig. 4. The most evident effect of doping is the appearance of new states: the occupied boron $p_{z}$ orbital at about $-2 \mathrm{eV}$ dispersing along $k_{\|}$, and the empty nitrogen $p_{z}$ orbital at about $+1.5 \mathrm{eV}$, showing a smaller dispersion. Four other states appear in the projected-DOS gap. These states have one-dimensional character and are fully localized in the scattering region. For this reason, they do not contribute to the electronic transport but they behave as scattering centers.

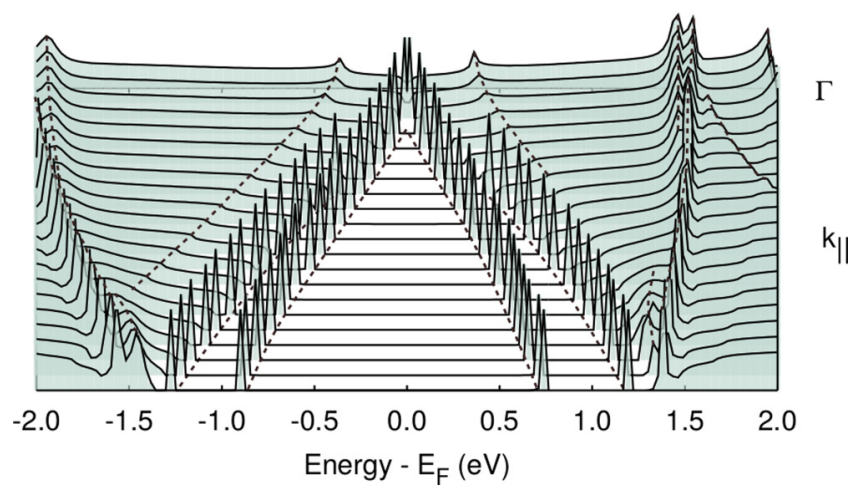

FIG. 4. $k_{\|}$-resolved DOS of the overlay nanojunction shown in Fig. 3.
We calculated the $I(V)$ curve with the NEGF-DFT method, where the charge density is self-consistently updated upon the effect of the applied bias. Once the transmission function is worked out, the current is obtained, integrating the transmission probability into the energy window corresponding to the applied voltage:

$$
I(V)=\frac{e}{h} \int d E T(E, V)\left[f_{L}(E)-f_{R}(E)\right],
$$

where $T(E, V)$ is the transmission function and $f_{L / R}(E)$ represents the occupation function of the $L / R$ electrode [22].

We report in Fig. 5 the $I(V)$ curves for the doped and pristine BLG. The comparison shows that the current flowing in the B-N-doped system is only slightly larger than that in pristine BLG. Indeed, the $\mathrm{N}$ and $\mathrm{B}$ states do not perturb the system in the energy range relevant for transport and the $\pi$ electrons' delocalization in the carbon network is preserved.

As an example, in the inset of Fig. 5, the transmission function for these two systems at an applied voltage of $-1.5 \mathrm{~V}$ is reported. They look very similar, especially in the bias energy window, further confirming the previous analysis. At the applied voltages considered, the current in the pristine BLG amounts to twice that in a single sheet of graphene. This suggests that the interlayer current flow is negligible with respect to the intralayer one also in the presence of dopants.

In order to study the role played by the dopants on the interlayer current, we now consider a "shingle" nanojunction, differing from the overlay as the contact is made by overimposing the terminations of two semi-infinite $\mathrm{H}$ terminated graphene layers (Fig. 6). The contact region is hence constituted by partial overlap of doped graphene flakes. In the present case, the electron current must flow from one graphene layer to the other, overcoming an energy barrier. This system breaks the left-right symmetry and can behave like a p-n nanojunction.

The computed $I(V)$ characteristics are reported in Fig. 7, where positive biases indicate flow of electrons from the $\mathrm{N}$-doped layer to the B-doped one. The first finding is the

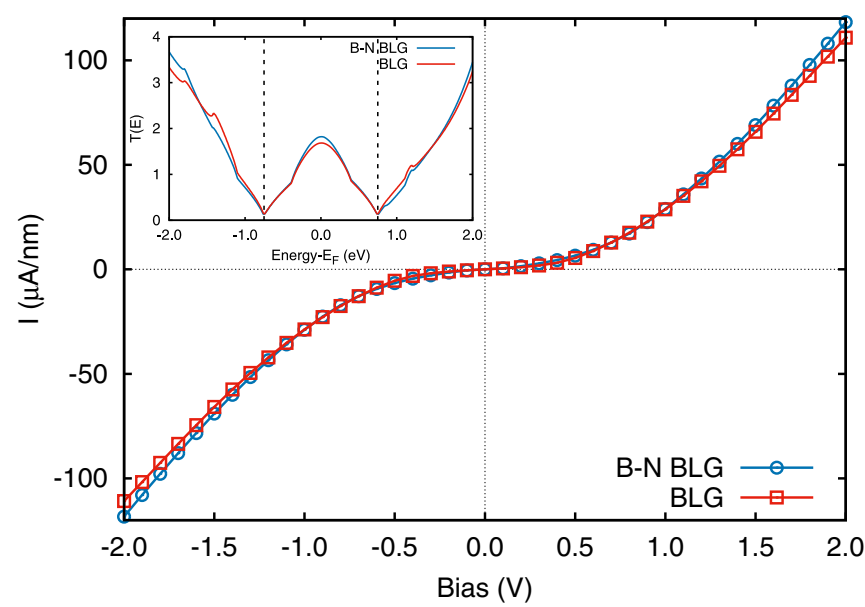

FIG. 5. $I(V)$ characteristic of the overlay nanojunction shown in Fig. 3 (blue circles) compared to that of pristine BLG (red squares). The inset reports the transmission probability at a bias potential of $-1.5 \mathrm{~V}$. 


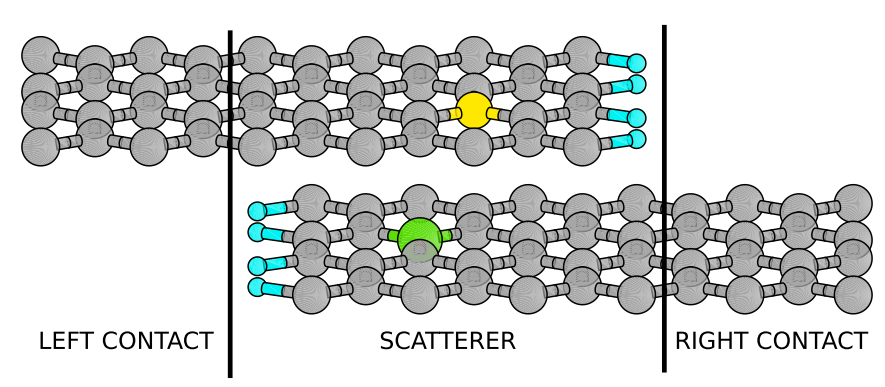

FIG. 6. Geometry of the B-N-doped shingle nanojunction in the $\mathbf{C}$ arrangement. The upper layer contains the nitrogen atom while the lower layer contains the boron one.

reduction of the absolute value of the current by almost one order of magnitude. This reduction does not completely suppress the current flow because of the partial overlap of the graphene $\pi$ bands on different layers. We note that for positive biases, the electron current through the doped junction is slightly larger than that obtained without the B-N pair.

The most interesting result is the behavior of the junction at large negative biases $(V<-1.0 \mathrm{~V})$ : in this case, the current of the doped system is significantly larger (about $50 \%$ ) than for the pristine one. Furthermore, the asymmetry of the junction produces different values for the current at positive/negative biases, giving rise to a rectification behavior. The transmission functions at $-1.5 \mathrm{~V}$ bias are reported in the inset of Fig. 7. The doped system is characterized by a larger value particularly evident in the middle of the bias energy window.

The shingle junction geometry hence shows a remarkable role played by the $\mathrm{BN}$ couple on the transport properties. As any real junction would also include possibly different structures, it is interesting to see how the other relative arrangements of the $\mathrm{B}$ and $\mathrm{N}$ atom would influence the

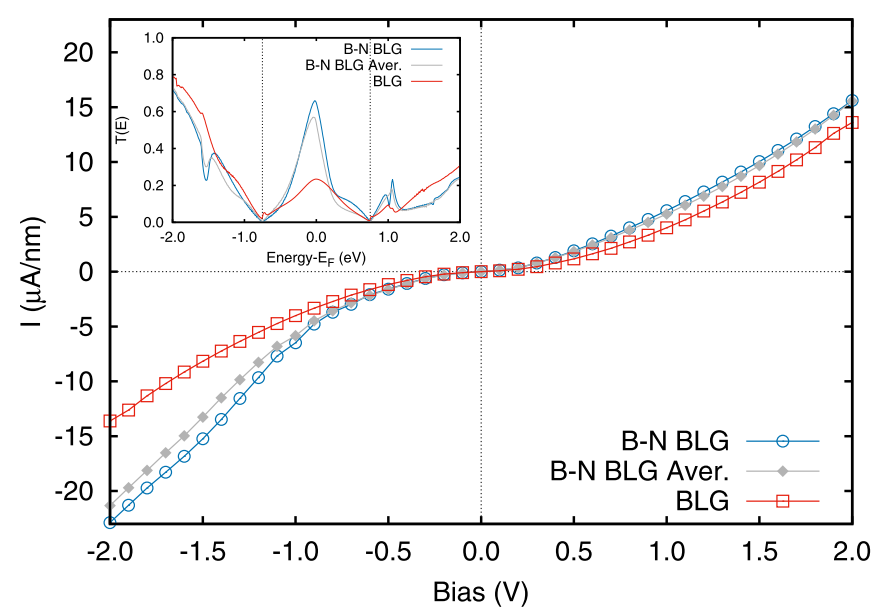

FIG. 7. $I(V)$ characteristic of the shingle nanojunction. In addition to results for the most stable $\mathbf{C}$ arrangement depicted in Fig. 6 (blue void circles) and those for pristine BLG (red void squares), we show those resulting from the average of arrangements $\mathbf{A}, \mathbf{B}$, and $\mathbf{C}$ modeling configurational disorder in the doping (gray filled squares). The inset reports the transmission probability at a bias potential of $-1.5 \mathrm{~V}$, where electron transport occurs from the B-doped layer to the $\mathrm{N}$-doped one.

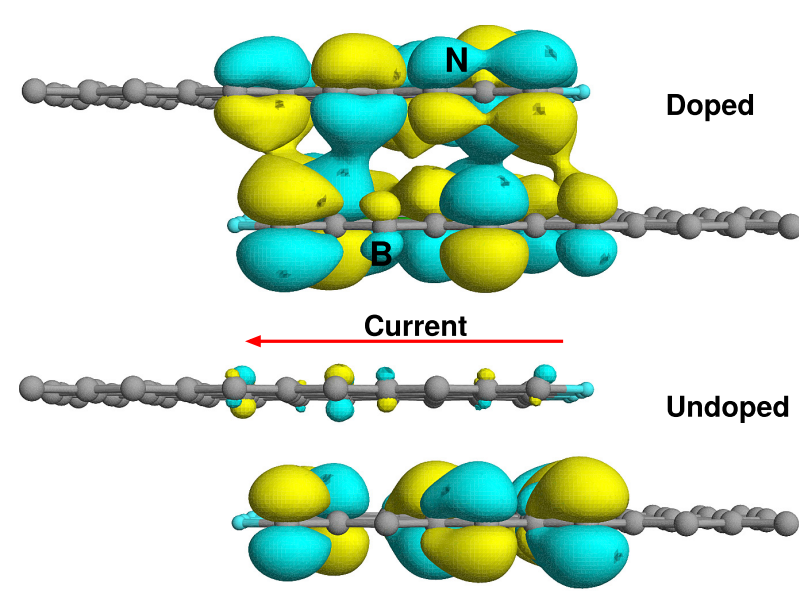

FIG. 8. Eigenchannels at $E=0 \mathrm{eV}$ and $k_{\|}=0.12 \AA^{-1}$ for the doped shingle nanojunction (upper panel) and for the corresponding undoped case (lower panel). The applied bias is $-1.5 \mathrm{~V}$.

transport. Here we can focus on the three relative arrangements (i.e., $\mathbf{A}, \mathbf{B}$, and $\mathbf{C}$ ), recalling that the electronic properties are only mildly dependent on the actual position of the dopants, once the sublattice is fixed. Since $\mathbf{A}$ and $\mathbf{B}$ are only slightly higher in energy $(\sim 0.06 \mathrm{eV}$ in the periodic case), we assume an equal occurrence of the three cases and report in Fig. 7 the $I(V)$ characteristic averaged over them. This demonstrates that the conclusions derived above for case $\mathbf{C}$ also apply to a more realistic case where configurational disorder and different relative B-N arrangements are present. Such an analysis is not interesting for the overlay junction case (see Fig. 3) where the $I(V)$ characteristic of BLG is retained also upon doping.

Through a more detailed inspection of the electronic properties relevant for the transport, we can rationalize the asymmetry in the current considering that the application of a bias tends to shift the electronic states of the dopants. In fact, for positive biases, the empty $\mathrm{N}$ state is further raised while the occupied B state is lowered. This effect does not change the transport properties in the relevant energy window around the Fermi level. Differently, for negative biases, these two states move towards the Fermi level, eventually entering in the bias windows for voltages $<-1.0 \mathrm{~V}$. The first state playing a role in transport is the empty $\mathrm{N}$ state for large $k_{\|}$ (see its dispersion in Fig. 4). For a spatial representation of this aspect, we report in Fig. 8 the eigenchannels [23] in the scattering region, obtained at $E=0 \mathrm{eV}$ and $k_{\|}=0.12 \AA^{-1}$ for both the doped and undoped shingle nanojunction. It is evident how the presence of the dopants allows the electrons to flow from one layer to the other. The transmission coefficients for these particular states are $t=0.95$ and $t=0.14$, respectively. It can be noted that the transmission channels do not directly connect the $\mathrm{B}$ and $\mathrm{N}$ atoms, but they instead joint the entire carbon lattices of the two layers. Therefore, the presence of these impurities plays the role to lower the effective barrier between layers.

In conclusion, we studied the effects of the B-N doping on the electronic and transport properties of the graphene bilayer by first principles. We found the opening of a gap 
amounting to $0.35 \mathrm{eV}$ with the highest occupied molecular orbital (HOMO) belonging to the B-doped layer and the lowest unoccupied molecular orbital (LUMO) to the N-doped layer. Furthermore, the band structure resembles that of a pristine graphene bilayer upon the application of a strong electric field. Our analysis showed a small effect of the dopants on the transport properties of the graphene bilayer. Differently, when the carbon network is broken as in overlapping graphene flakes, the dopants have a sizable effect on the interlayer current. In particular, for negative biases, the presence of the dopants enhances the current, originating a rectification effect.
[1] Y. Zhang, T.-T. Tang, C. Girit, Z. Hao, M. C. Martin, A. Zettl, M. F. Crommie, Y. R. Shen, and F. Wang, Nature (London) 459, 820 (2009).

[2] J. Nilsson, A. H. Castro Neto, F. Guinea, and N. M. R. Peres, Phys. Rev. Lett. 97, 266801 (2006).

[3] G. Fiori and G. Iannaccone, IEEE Electron Device Lett. 30, 1096 (2009).

[4] S. K. Banerjee, L. F. Register, E. Tutuc, D. Reddy, and A. H. MacDonald, IEEE Electron Device Lett. 30, 158 (2009).

[5] S.-L. Li, H. Miyazaki, H. Hiura, C. Liu, and K. Tsukagoshi, ACS Nano 5, 500 (2011).

[6] M. Lafkioti, B. Krauss, T. Lohmann, U. Zschieschang, H. Klauk, K. von Klitzing, and J. H. Smet, Nano Lett. 10, 1149 (2010).

[7] Z.-M. Liao, B.-H. Han, Y.-B. Zhou, and D.-P. Yu, J. Chem. Phys. 133, 044703 (2010).

[8] P. Ordejón, E. Artacho, and J. M. Soler, Phys. Rev. B 53, R10441(R) (1996).

[9] J. M. Soler, E. Artacho, J. D. Gale, A. García, J. Junquera, P. Ordejón, and D. V. Sánchez-Portal, J. Phys. Condens. Matter 14, 2745 (2002).

[10] E. Artacho, E. Anglada, O. Dieguez, J. D. Gale, A. García, J. Junquera, R. M. Martin, P. Ordejón, J. M. Pruneda, D. V. Sánchez-Portal, and J. M. Soler, J. Phys. Condens. Matter 20, 064208 (2008).
[11] E. Artacho, D. V. Sánchez-Portal, P. Ordejón, A. García, and J. M. Soler, Phys. Stat. Sol. B 215, 809 (1999).

[12] N. Troullier and J. L. Martins, Phys. Rev. B 43, 1993 (1991).

[13] J. P. Perdew, K. Burke, and M. Ernzerhof, Phys. Rev. Lett. 77, 3865 (1996).

[14] S. Grimme, J. Comput. Chem. 27, 1787 (2006).

[15] S.-M. Paek, E. Yoo, and I. Honma, Nano Lett. 9, 72 (2009).

[16] A. L. Vázquez de Parga, F. Calleja, B. Borca, M. C. G. Passeggi, J. J. Hinarejos, F. Guinea, and R. Miranda, Phys. Rev. Lett. 100, 056807 (2008).

[17] E. V. Castro, K. S. Novoselov, S. V. Morozov, N. M. R. Peres, J. M. B. Lopes dos Santos, J. Nilsson, F. Guinea, A. K. Geim, and A. H. Castro Neto, Phys. Rev. Lett. 99, 216802 (2007).

[18] C.-H. Park, F. Giustino, M. L. Cohen, and S. G. Louie, Nano Lett. 8, 4229 (2008).

[19] A. Lherbier, X. Blase, Y.-M. Niquet, F. Triozon, and S. Roche, Phys. Rev. Lett. 101, 036808 (2008).

[20] M. Brandbyge, J. L. Mozos, P. Ordejón, J. Taylor, and K. Stokbro, Phys. Rev. B 65, 165401 (2002).

[21] S. Datta, Electronic Transport in Mesoscopic Systems (Cambridge University Press, Cambridge, 1995).

[22] S. Datta, Quantum Transport: Atom to Transistor (Cambridge University Press, Cambridge, 2005).

[23] M. Paulsson and M. Brandbyge, Phys. Rev. B 76, 115117 (2007). 